\title{
Management of childhood urinary tract infection
}

Weerasooriya $W$ A L K $\boldsymbol{K}^{1}$

${ }^{1}$ Teaching Hospital Jaffna.

\begin{abstract}
One of the common infections among children is urinary tract infection. To reduce the morbidity associated with UTI, it is very important to diagnose promptly and initiate correct management. Signs and symptoms vary among different age groups, as well as some clinical features are nonspecific. Hense, accurate diagnosis is challenging and which is masked further by wide use of antibiotic prior to the collection of urine for culture and full report. Use of correct antibiotic for appropriate duration is crucial in management. Ideal image protocol in subsequent follow-up is still not very clear and debatable among majority.
\end{abstract}

\section{Key words}

Children, urinary tract infection, management

\section{Introduction}

One of the common infections among children is urinary tract infection. Signs and symptoms vary among different age groups, according to associated uropathies, as well as those are nonspecific. Since young infants have non-specific symptoms, a high index of suspicion is needed to consider a clinical diagnosis of UTI. All children with unexplained fever $>38 \mathrm{C}$, all children with symptoms and signs suggestive of UTI and children with an alternative site of infection, who remain unwell after treatment have to bescreened for UTI.

Upper urinary tract infection is defined as infections involving the renal parenchyma causing systemic and local symptoms. Lower urinary tract infection defined as infection limited to lower urinary tract mainly leading to voiding symptoms. Recurrent UTI is defined as presence of $\geq 2$ episodes of upper UTI OR 1 episode of upper UTI plus lower UTI $\geq 1$ episodes OR $\geq 3$ episodes of lower UTI $(1,2)$. Atypical or complicated UTI define as UTI associated with anatomical or functional ab- normalities of the urinary tract or resulting raised serum creatinine, sepsis, poor urine flow, abdominal mass and failure to respond to treatment with the appropriate antibiotic within $48 \mathrm{hrs}$.

\section{Clinical features}

The evaluation of a child should include a through history and physical examination. Diagnosis of UTI among children is rarely straight forward. Identification of children at risk of recurrent infections (Eg; labial adhesion, constipation, tight prepuce, voiding dysfunction, neurogenic bladder) is also important in appropriate management. (2)

- Children $<3$ month - present commonly with fever, vomiting, lethargy, irritability and less commonly poor feeding, failure to thrive and offensive urine

- Children 3months to infancy - present with fever, abdominal pain, loin tenderness, vomiting and poor feeding

- Among older children - Frequency, dysuria, lower urinary tract symptoms (LUTS), abdominal pain, loin tenderness and fever.

Presence of macroscopic haematuria also may be a symptom of UTI among any age group.

\section{Pathogens}

$E$ - coli is accountable for $80-90-\%$ of community acquired urinary tract infection in children. Proteous mirabilis, Staphylococcus saprophyticus and Klebsiella species represent the rest as less common pathogens $(3,4)$.

\section{Diagnosis}

Presence of bacteria in sufficient quantity, with concurrent evidence of an on-going infection suggests UTI . Both urine culture and microscopy are needed for the accurate diagnosis of urinary tract

Corresponding author: Weerasooriya W A L K, email: Weerasooriya.lk@gmail.com, (D) https://orcid.org/0000-0003-3380-5975, Submitted June2020, Accepted October 2020 
infection. Urine culture is the goal standard for UTI diagnosis ( 4 ). Positive urine culture with normal urine analysis in asymptomatic, otherwise healthy child is considered as asymptomatic bacteriuria do not need intervention or treatment.

\section{Investigations}

Inflammation due to UTI is well indicated by urine microscopic assessment. Positive urine analysis is considered when presence of $>10 \mathrm{WBC} / \mu 1$ in uncentrifuged sample asses by microscopy or $>5$ WBC in centrifuged sample ( 5 ).

Urine sample is collected for culture as either Clean Catch Mid Stream Urine (CCMSU) sample, in out catheter demonstrates a single uropathogen in quantity of $10^{5} / \mathrm{ml}$ or more is considered as positive culture $(1,2)$. However, threshold may be varied according to the method of collection. Any growth of gram negative bacilli or more than $10^{3}$ / $\mathrm{ml}$ of gram positive cocci on supra pubic sample is considered as positive $(1,2)$. Furthermore, if urine analysis and clinical picture is highly suggestive and growth of $10^{4-5} / \mathrm{ml}$ also may be considered as positive sample $(1,2)$. Collection of urine sample for culture via suprapubic aspiration is recommended when there is inability to obtain a catheter sample when the urethra is not visualized in a boy with very tight prepuce or a girl with severe labial adhesions or presence of gross infection around genitalia.

\section{Treatment}

Maintaining adequate hydration is important during the management. Child who is $<3$ month, severely ill, having complex underline urogenital anomaly and cannot retain oral drugs, initial intravenous route is recommended. All patients should be reassessed after 48 hours of starting antibiotics, and treatment should be reviewed as per cultures and sensitivities.

- Lower UTI or cystitis should be treated for 7 days. Recommended drugs for empirical treatment are nitrofurantoin, nalidixic acid, cephalexin and co-amoxiclav.

- Uncomplicated upper UTI should be treated for 7 - 14 days. Recommended drugs for empirical treatment are cephalexin, co-amoxiclav, cefuroxime.

\section{Follow up}

USS KUB is indicated for all children following an attack of UTI within 6 weeks. USS KUB is indicated during acute infection for those who have recurrent UTI (if $<6$ months of age) and atypical UTI despite of age $(1,2,6)$.

DMSA is indicated only for children with atypical UTI ( < 3years of age), recurrent UTI (all age groups) and USS evidence of significant renal parenchymal defects or damage (all age group) $(1,2,6)$.

MCUG is indicated for those who are suspected with bladder outlet obstruction (all age groups) and atypical or recurrent UTI ( $<6$ months of age) $(1,2,6)$.

\section{Prophylactic antibiotics}

Prophylactic antibiotics are not routinely recommended for children following first UTI and also those with isolated low grade VUR, isolated renal scarring, since this practice can lead to multi drug resistance $(1,2,7)$. However there are some instances where children might be benefited with giving prophylaxis such as major structural abnormalities of KUB including grade IV - V VUR, recurrent UTI, bladder bowel dysfunction (BBD), children with high risk of renal damage and not having free access to health care facilities during acute episodes.

\section{Complication and prognosis}

There is risk of damage to renal parenchyma and lead to scaring due to the infection causing inflammatory reaction. As a consequences, it will result in hypertension and renal impairment in long term. (8).

\section{Conclusion}

Urinary tract infection is a common infection among children. Diagnosis can be challenging but is important to consider, especially for infants with fever without focus. Prompt and accurate diagnosis, appropriate management is important for reduce the short as well as long-term morbidity. Management should be aimed at treating the current episode, recognise the aetiology and preventing recurrences. Collection of sterile urine sample is fundamental for diagnosis. Recent evidence supports less aggressive imaging following uncomplicated UTI. 
Further research is required to improve some gaps and cost-effective care for children with UTI.

\section{Abbreviations and symbols:}

UTI - Urinary tract infection,LUTS - Lower urinary tract symptoms, CCMSU - Clean catch midstream urine,USS KUB - Ultrasound scan Kidney, Ureter, Bladder,DMSA - Dimercaptosuccinic acid,MCUG-Micturating cystourethrogram,VUR - Vesico ureteric reflux ,BBD - Bladder bowel dysfunction.

\section{References}

1. National Institute for Health and Clinical Excellence: Urinary tract infection in children London: NICE,2007

2. Mc Taggart S, Danchin M, Ditch field M, et al. KHA-CARI summary guideline: Diagnosis and treatment of urinary tract infection in children. South Melbourne, Vic: Kidney Health Australia,2014

3. Okarska-Napierała M, WasilewskaA, Kuchar E. Urinary tract infection in children: Diagnosis, treatment, imaging-Comparison of current guidelines. Journal of pediatric urology. 2017 Dec 1;13(6):567-73.American Academy of Paediatrics Practice Parameter.
The diagnosis, treatment and evaluation of the initial urinary tract infection in febrile and young children. Pediatrics 1999; 103:843-852

4. Kaufman J, Temple-Smith M, Sanci L. Urinary tract infections in children: an overview of diagnosis and management. BMJ paediatrics open. 2019;3(1).

5. Doern CD, Richardson SE. Diagnosis of urinary tract infections in children. Journal of clinical microbiology. $2016 \mathrm{Sep}$ 1;54(9):2233-42. doi:10.1128/JCM.0018916

6. Paintsil E. Update on recent guidelines for the management of urinary tract infections in children: the shifting paradigm. Current opinion in pediatrics. $2013 \mathrm{Feb} ; 25(1): 88$

7. Jacobson SH, Eklof O, Eriksson CG, Lins LE, TidgrenB, Winberg J. Development of hypertension and uraemiaafter pyelonephritis in childhood: 27 year follow up. BMJ.1989;299:703---6

8. Zorc JJ, Kiddoo DA, Shaw KN. Diagnosis and management of pediatric urinary tract infections. Clinical microbiology reviews. 2005 Apr 1;18(2):417-22. 